\title{
Highlights from the CMS Experiment
}

\author{
Juan Alcaraz Maestre ${ }^{* \dagger}$ \\ Particle Physics Division \\ Center for Energy, Environment and Technology Research (CIEMAT) \\ Avda. Complutense, 40, 28040 - Madrid \\ E-mail: Juan.Alcaraz@ciemat.es
}

We discuss the status and some recent results of the CMS experiment at the LHC. The performance of the detector is assessed using a luminosity of $\approx 5 \mathrm{fb}^{-1}$ recorded in the first part of the 2017 data-taking period. Run 2 physics studies use data collected at a center-of-mass energy of $\sqrt{s}=13 \mathrm{TeV}$, corresponding to an integrated luminosity of $36 \mathrm{fb}^{-1}$. Highlights from studies in the Higgs sector are the first observation of its decay into tau leptons by a single experiment, the most precise measurement of its mass, $\mathrm{m}_{\mathrm{H}}=125.26 \pm 0.21 \mathrm{GeV}$ and a first search for the $\mathrm{H} \rightarrow \mathrm{b} \overline{\mathrm{b}}$ decay in a phase space region that is sensitive to the gluon-fusion production mechanism. In the electroweak sector, CMS provides the currently most precise measurement of the effective weak mixing angle at the LHC: $\sin \theta_{\mathrm{eff}}^{\text {lept }}=0.23101 \pm 0.00052$, using an integrated luminosity of $\approx 20 \mathrm{fb}^{-1}$ at $\sqrt{s}=8 \mathrm{TeV}$. Other CMS highlights of this conference are new stringent limits on di-Higgs production and improved searches for dark matter and electroweakinos.

The European Physical Society Conference on High Energy Physics

5-12 July, 2017

Venice

\footnotetext{
* Speaker.

On behalf of the CMS Collaboration.
} 


\section{Status of 2017 data-taking and recent upgrades}

CMS [1] underwent several upgrades during the last 2016-2017 Extended Year-End Technical Stop (EYETS). A major modification was the installation of a new four-layer pixel detector, aiming for improved b-tagging and vertex identification capabilities near the interaction point. The Phase 1 Upgrade of the Hadron Calorimeter was partially completed. It involved the full deployment of the new front-end read-out of the Hadron Forward calorimeter and one new read-out sector of the Hadron End-cap calorimeter, spanning $20^{\circ}$ in the azimuthal coordinate. Five slices of the future Phase 2 GEM GE1 detector, covering $10^{\circ}$ each were also installed and are being tested and operated now. More details on the current status of the CMS upgrades can be found in [2] and references therein.

The first layer of the new CMS Barrel Pixel detector [3] is extremely close to the beam line ( $3 \mathrm{~cm}$ radius). It has a special read-out chip in order to cope with the rates expected at LHC for instantaneous luminosities of order $210^{34} \mathrm{cms}^{-2} \mathrm{~s}^{-1}$ and above. A different readout speed required a redefinition of its timing settings, something that was implemented and optimized using the first data collected in 2017. The performance of the new pixel detector was also studied with these data. The high-level trigger matching efficiency was found to be $\approx 97 \%$ for tracks traversing four pixel layers, while track residuals were found to be in agreement with the expectations for this early alignment phase. B-tagging variables, defined using a first (prompt) version of the reconstruction and preliminary alignment parameters show performances at the level of those of 2016 or slightly better. This is illustrated in Figure 1-left, which shows the distribution of the number of secondary vertices for jets with transverse momentum above $50 \mathrm{GeV}$.

CMS has recorded a luminosity of $5.3 \mathrm{fb}^{-1}$ at $\sqrt{s}=13 \mathrm{TeV}$ since the start of the 2017 data taking period. A fraction of these initial data was used to commission the new pixel detector. The operational efficiency of the CMS detector is excellent, with a $>95 \%$ fraction of active channels in all sub-detectors (Figure 1-right). The quality of lepton identification is already optimal at the current stage. The relative energy scale in the electromagnetic calorimeter, as measured using $\pi^{0}$ reference samples shows no bias and a stability at the $0.2 \%$ level (Figure 2-top). New Level-1 calorimeter trigger algorithms led to significant gains in the quality and thresholds of electron/photon triggers. The transverse momentum threshold for single muon triggers is still kept at $25 \mathrm{GeV}$, thanks to further improvements in the L1 muon trigger system. Muon performance at high momentum is also close to optimal at this early stage. Figure 2-bottom shows the most massive dimuon candidate ever collected in CMS $(2.4 \mathrm{TeV})$.

\section{Recent physics results from CMS}

The CMS collaboration has published more than 600 physics papers to date, being the LHC experiment with the largest number of submitted papers (to arXiv) in the last year. Substantial efforts were made to process and analyze the full 2016 data sample in a timely way. Data were reprocessed last Fall using optimal alignment and calibration constants, as well as the latest improvements in tracker reconstruction. A special computing campaign was carried out over Christmas time and about $10^{9}$ Monte Carlo events were made available for early 2017 analyses. CMS presented about 60 new results at Winter/Spring conferences, and $\approx 20$ additional results were released for this 

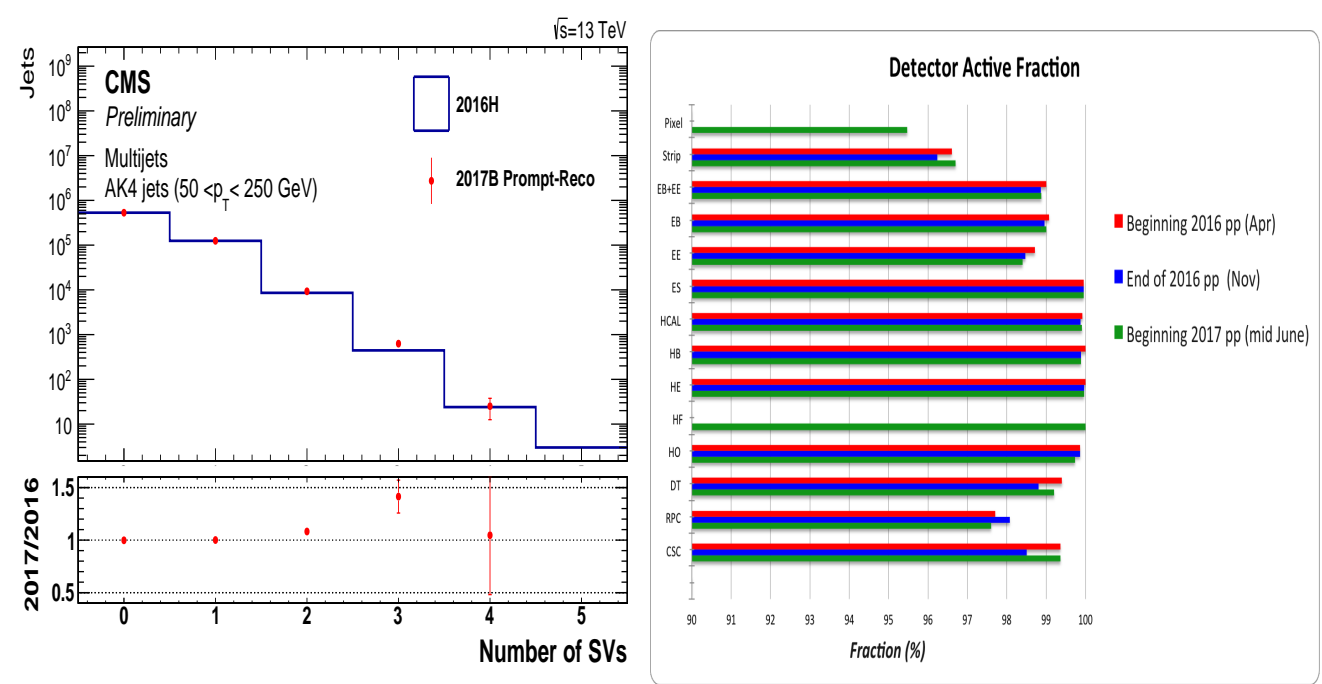

Figure 1: Left: distribution of the number of secondary vertices reconstructed in CMS data. The figure compares the performances of 2016 and 2017 configurations using multijet selected events. Only jets with transverse momentum in the $50<p_{\mathrm{T}}<250 \mathrm{GeV}$ range are considered. The performance of the new detector is found to be similar or slightly better than the one corresponding to the old pixel detector. The performance of last year data was evaluated in the last data-taking period $(2016 \mathrm{H})$, using the most recent versions of the reconstruction program and alignment parameters. The response of new detector was studied in early data (2017B), with first alignment parameters and an initial version of the reconstruction program (Prompt-Reco). Right: fraction of CMS active channels in 2017, compared with the status at the beginning and the end of the 2016 data taking period (taken from https://cern.ch/twiki/bin/view/CMSPublic/ActiveChannelsSummary).

HEP-EPS conference. It is not possible to summarize in this short report all these new results, so we refer the interested reader to other contributions to this conference for details on those analyses. In this summary we highlight the most recent advances in the Higgs, top and electroweak sectors, as well as the latest searches for supersymmetry and dark matter.

\subsection{Higgs Physics Results}

CMS has performed detailed studies of the intrinsic properties and production mechanisms of of the Higgs boson at $\sqrt{s}=13 \mathrm{TeV}$. Some of the most precise studies were done in the $\mathrm{H} \rightarrow \mathrm{ZZ}^{*} \rightarrow$ $4 \ell$ channel [6]. A clear Higgs peak in the four-lepton mass distribution is observed (Figure 3-left), corresponding to a fiducial cross section in the accessible experimental volume [6] of: $\sigma_{\text {fid }}=$ $2.92_{-0.44}^{+0.48}$ (stat. $)_{-0.24}^{+0.28}$ (syst.) fb. This result is in agreement with a standard model prediction of $2.76 \pm 0.14 \mathrm{fb}$. Figure 3-right shows the evolution of the measured fiducial cross section as a function of the center-of-mass energy.

Matrix element calculations and full kinematic information is used to define several kinematic discriminators. These discriminators are essential to disentangle the different Higgs production mechanisms: gluon fusion, associated weak boson production and vector-boson fusion. CMS thus obtains precise measurements of the different signal strengths relative to the standard model expectations, as well as direct limits on the Higgs width and the currently world best measurement of the Higgs mass: $m_{H}=125.26 \pm 0.20$ (stat.) \pm 0.081 (syst.) $\mathrm{GeV}$, still dominated by statistical un- 

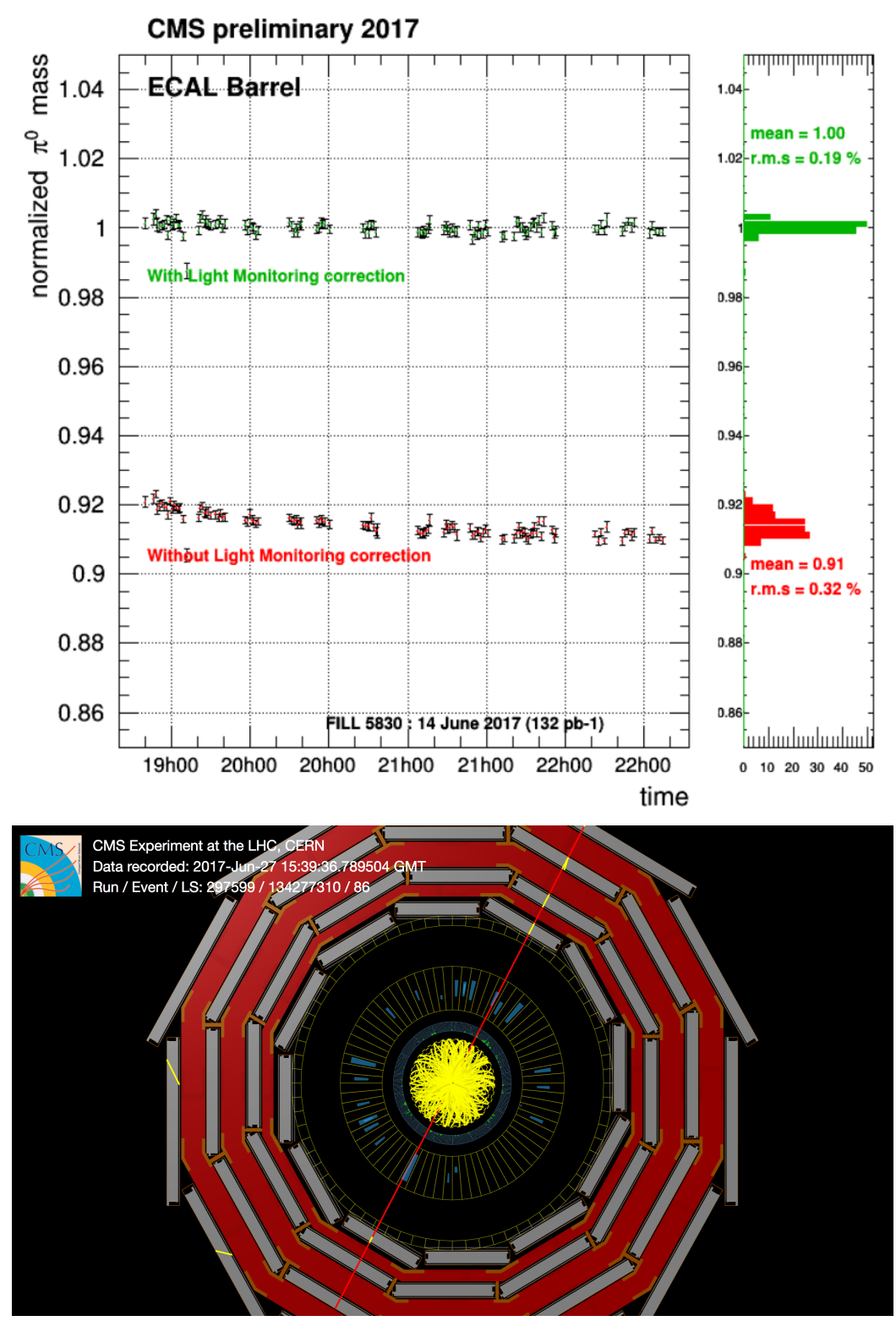

Figure 2: Top: scale bias and stability of the energy measured with the CMS electromagnetic calorimeter in 2017, evaluated on a $\pi^{0}$ calibration sample [4]. Bottom: most massive dimuon event (2.4 TeV mass) collected by the CMS detector $(2017,[5])$. 

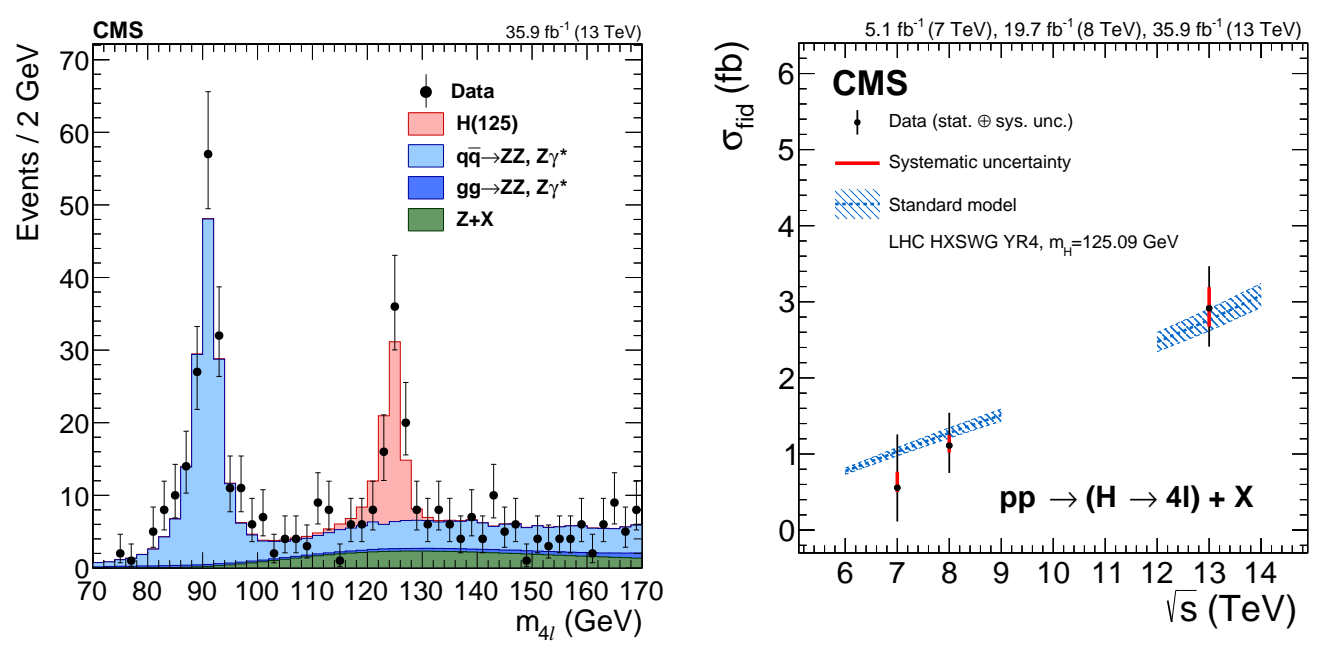

Figure 3: Results from the CMS analysis of the $\mathrm{H} \rightarrow \mathrm{ZZ}^{*} \rightarrow 4 \ell$ decay channel [6]. Left: Four-lepton invariant mass distribution; right: fiducial cross section measurements as a function of the center-of-mass energy, compared with theoretical expectations [7].

certainties. The low systematic uncertainty reflects a good understanding of the energy-momentum detector response for electrons and muons. The analysis [6] is completed with a comprehensive study of differential distributions as a function of the Higgs transverse momentum and the characteristics of the associated jet environment. A separate analysis [8] is used to test alternative spinparity hypotheses of the structure of the Higgs decay. No deviations with respect to a pure scalar $\left(0^{+}\right)$decay scenario are observed, leading to stringent limits on possible anomalous couplings.

The CMS Collaboration has presented at this conference what can be considered to be the first observation of the $\mathrm{H} \rightarrow \tau^{+} \tau^{-}$decay by a single experiment [9], and an essential step in the understanding of the fermionic (and leptonic) Yukawa terms predicted in the standard model. The analysis of 2016 data relies on reasonably low tau-trigger thresholds and on the discriminating power of the reconstructed (full) invariant mass of distributions of $\tau \tau$ candidates against the dominant $\mathrm{Z} \rightarrow \tau \tau$ background. The different production mechanisms are statistically disentangled via simple categorization, in terms of the jet content and different tau decay modes. Figure 4-left shows the distribution of the combined $-\log _{10}(($ Signal + Background $) /$ Signal $)$ discriminant, built using the contributions of all bins in all sub-categories. A clear excess in the signal-dominated region is seen. One should note that purely hadronic tau decays have a significant weight in the final results, which are in nice agreement with a Higgs mass value of $125 \mathrm{GeV}$. The combination of $\sqrt{s}=8 \mathrm{TeV}$ and $\sqrt{s}=13 \mathrm{TeV}$ CMS results provides an observed (and expected) significance of 5.6 standard deviations [9].

The intensive development of tools to analyze boosted hadronic topologies in recent years has allowed significant advances in the search for new physics involving hadronic decays of $\mathrm{W}, \mathrm{Z}$, top and Higgs bosons. An important example is the first sensitive search for $\mathrm{H} \rightarrow \mathrm{b} \overline{\mathrm{b}}$ production via gluon-fusion performed by CMS, targeting boosted Higgs decays and an effective rejection of the huge QCD backgrounds [10]. The current luminosity collected at $\sqrt{s}=13 \mathrm{TeV}$ allows an estimate 
of the relative signal strength of this process of $\mu_{\mathrm{H}}=2.3 \pm 1.5$ (stat. $)_{-0.4}^{+1.0}$ (syst.), i.e. a tiny but promising significance at the $1 \sigma$ level. A by-product of the analysis is the establishment of $\mathrm{Z} \rightarrow \mathrm{b} \overline{\mathrm{b}}$ production with an observed (expected) significance of 5.1(5.8) standard deviations. Figure 4right summarizes these results in the plane of $\mathrm{H}, \mathrm{Z}$ signal strengths $\left(\mu_{\mathrm{Z}}\right.$ versus $\mu_{\mathrm{H}}$ ) relative to the standard model expectations. Finally, CMS has released most stringent limits on double Higgs production using the $\mathrm{HH} \rightarrow \gamma \gamma \mathrm{b} \overline{\mathrm{b}}$ channel [11]. A cross section of 19 times the standard model predictions is excluded (for an expected exclusion of 17 times the standard model) assuming a standard structure in production and decay. This is good news for the HL-LHC program, where an integrated luminosity of $\approx 100$ times the current Run 2 luminosity should provide the first hints of di-Higgs production, even in the context of no deviations from the standard model.
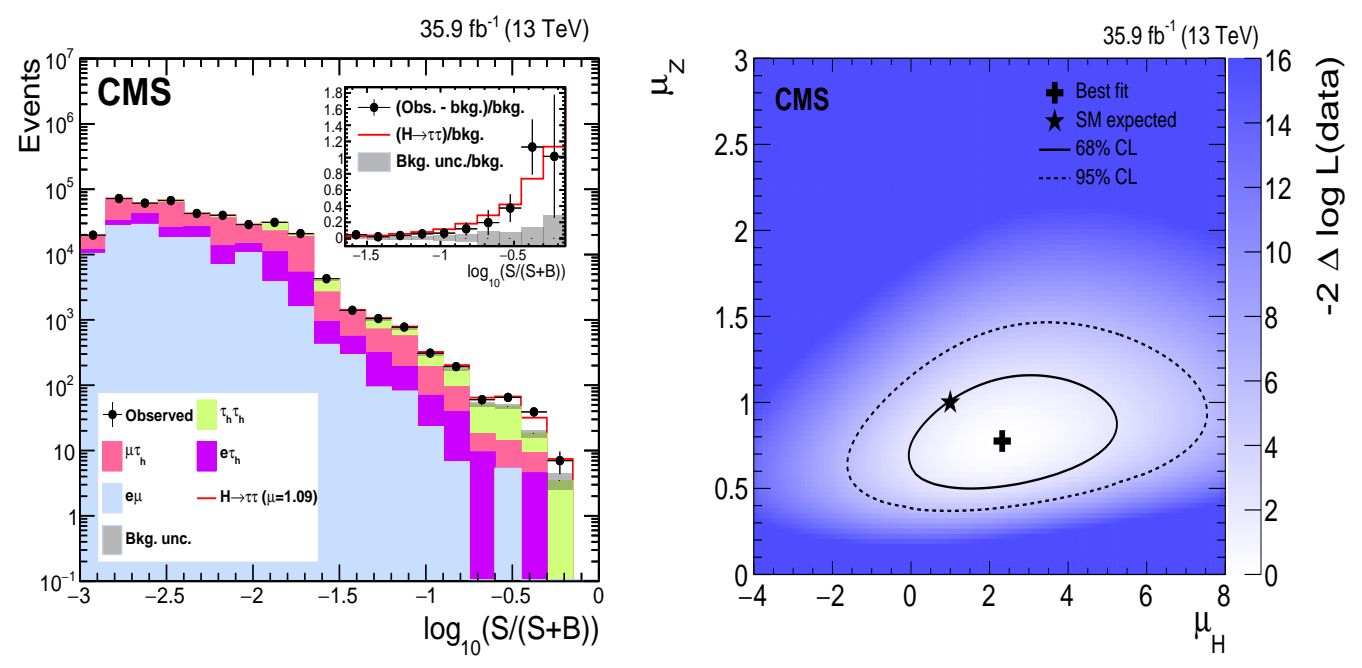

Figure 4: CMS searches for Higgs decays into fermions of the third generation at $\sqrt{s}=13 \mathrm{TeV}$. Left: Statistical combination of the $(S+B) / B$ contributions from all bins and sub-categories participating in the $\mathrm{H} \rightarrow \tau \tau$ search [9], showing a clear excess in the signal region. Right: combined measurements of the $\mathrm{H} \rightarrow \mathrm{b} \overline{\mathrm{b}}$ and $\mathrm{Z} \rightarrow \mathrm{b} \overline{\mathrm{b}}$ signal strengths relative to the standard model $\left(\mu_{\mathrm{Z}}\right.$ versus $\left.\mu_{\mathrm{H}}\right)$ in the CMS highly

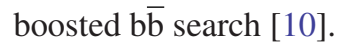

\subsection{Electroweak measurements}

Same-sign WW electroweak production is explored in CMS [12] by selecting events with two leptons (electrons or muons) of the same charge, moderate missing transverse energy and two jets of high momentum, separated by a large gap in pseudo-rapidity. The signal cross section is defined by association to the gauge-invariant set of diagrams that involve the virtual exchange of at least one electroweak boson. With this definition electroweak WW production is predicted to dominate the region of high values of the dijet invariant mass, as experimentally confirmed (Figure 5-left). The study provides for the first time at the LHC a clean observation of this production mechanism, with an observed (expected) significance of 5.5(5.7) standard deviations, as well as stringent limits on dimension-8 quartic couplings.

CMS has presented at this conference a preliminary measurement of the electroweak mixing angle, $\sin \theta_{\text {eff }}^{\text {lept }}$, using a luminosity of $\approx 20 \mathrm{pb}^{-1}$ collected at $\sqrt{s}=8 \mathrm{TeV}$ [13]. The parameter 
$\sin \theta_{\text {eff }}^{\text {lept }}$ denotes the mixing angle associated to the effective $\mathrm{Z} \rightarrow \ell^{+} \ell^{-}$coupling, while the experimental observable directly measured in the analysis is the forward-backward asymmetry of $\mathrm{Z} \rightarrow \mathrm{e}^{+} \mathrm{e}^{-}$and $\rightarrow \mu^{+} \mu^{-}$events, $\mathrm{A}_{\mathrm{FB}}$. The experimental definition of this asymmetry assumes that the direction of the quark initiating the hard process is associated to the beam direction that is closest to the one of the $\mathrm{Z}$ system. $\mathrm{A}_{\mathrm{FB}}$ is measured in multiple bins as a function of dilepton mass and rapidity. The uncertainty due to intrinsic parton density function (PDF) uncertainties is significantly reduced by imposing consistency of the derived values of $\sin \theta_{\text {eff }}^{\text {lept }}$ in all bins. The final result is the currently most precise measurement of this electroweak parameter at the LHC: $\sin \theta_{\text {eff }}^{\text {lept }}=0.23101 \pm 0.00036$ (stat.) \pm 0.00018 (syst.) \pm 0.00016 (theo.) \pm 0.00030 (PDF) $=$ $0.23101 \pm 0.00052$. Let us note that the measurement is still dominated by statistical uncertainties and PDF uncertainties could be further improved with more data within the adopted strategy. Figure 5-right illustrates the interplay between PDF uncertainties and measurements of the forward-backward asymmetry for different values of the mixing angle. Figure 6 shows the new CMS measurement compared with other measurements of the electroweak mixing angle at hadron and $\mathrm{e}^{+} \mathrm{e}^{-}$colliders.
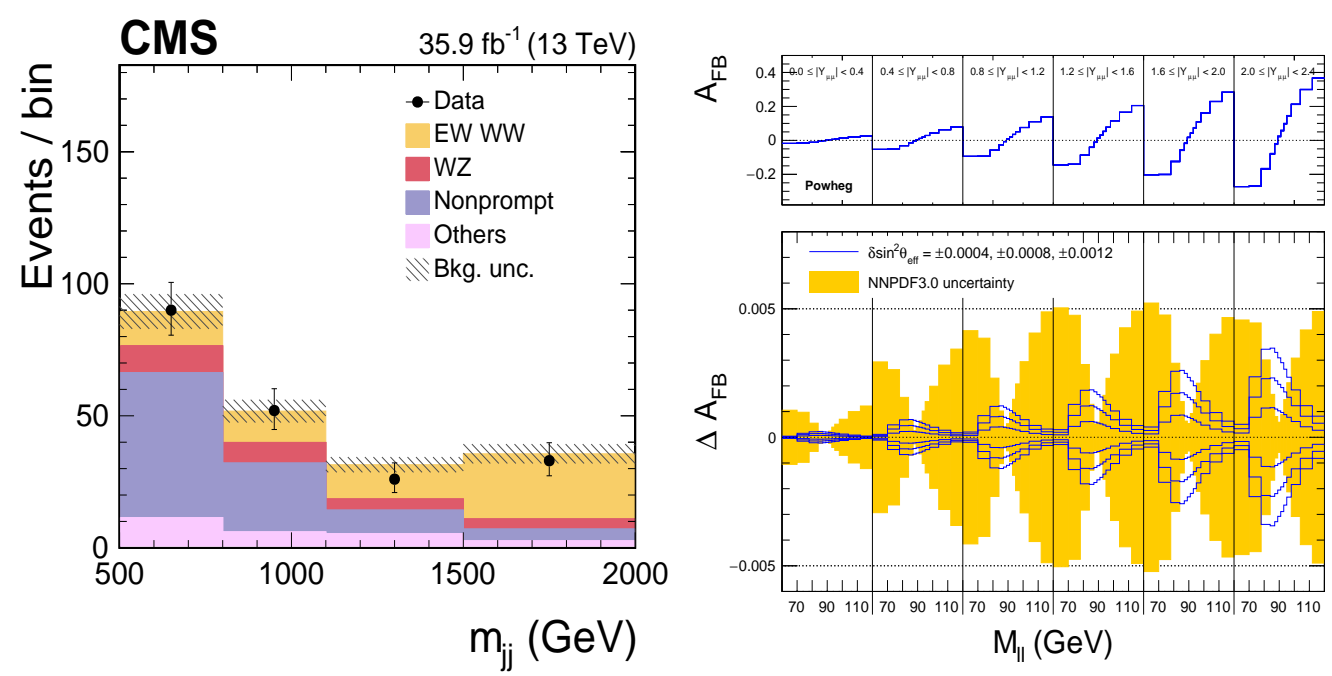

Figure 5: Left: observation of electroweak production of same-sign W pairs (EW WW) by CMS [12]. The plot shows the dijet mass distribution of selected events. Right: measurements of the forward-backward asymmetry in $\mathrm{Z} \rightarrow \ell^{+} \ell^{-}$events in CMS [13]. The figure illustrates the relation between PDF uncertainties and different possible values of the electroweak mixing angle around a central value of $\sin \theta_{\mathrm{eff}}^{\text {lept }}=0.23120$. The CMS measurement is performed in multiple bins of dilepton invariant mass and rapidity.

\subsection{Searches for new physics}

CMS has released in 2017 a new study of the angular distributions in $\mathrm{B}^{0} \rightarrow \mathrm{K}^{* 0} \mu \mu$ decays at $\sqrt{s}=8 \mathrm{TeV}$ [14]. It extends a previous one [15] by including the measurement of the coefficients known as P1 and P5'. The extension required the analysis of the differential distributions as a function of the azimuthal angle between the $\mathrm{K}^{* 0} \rightarrow \mathrm{K}^{+} \pi^{-}$and $\mu \mu$ decay planes (in the $\mathrm{B}^{0}$ rest frame). Figure 7 shows the measured evolution of the P5' parameter as a function of the square of 


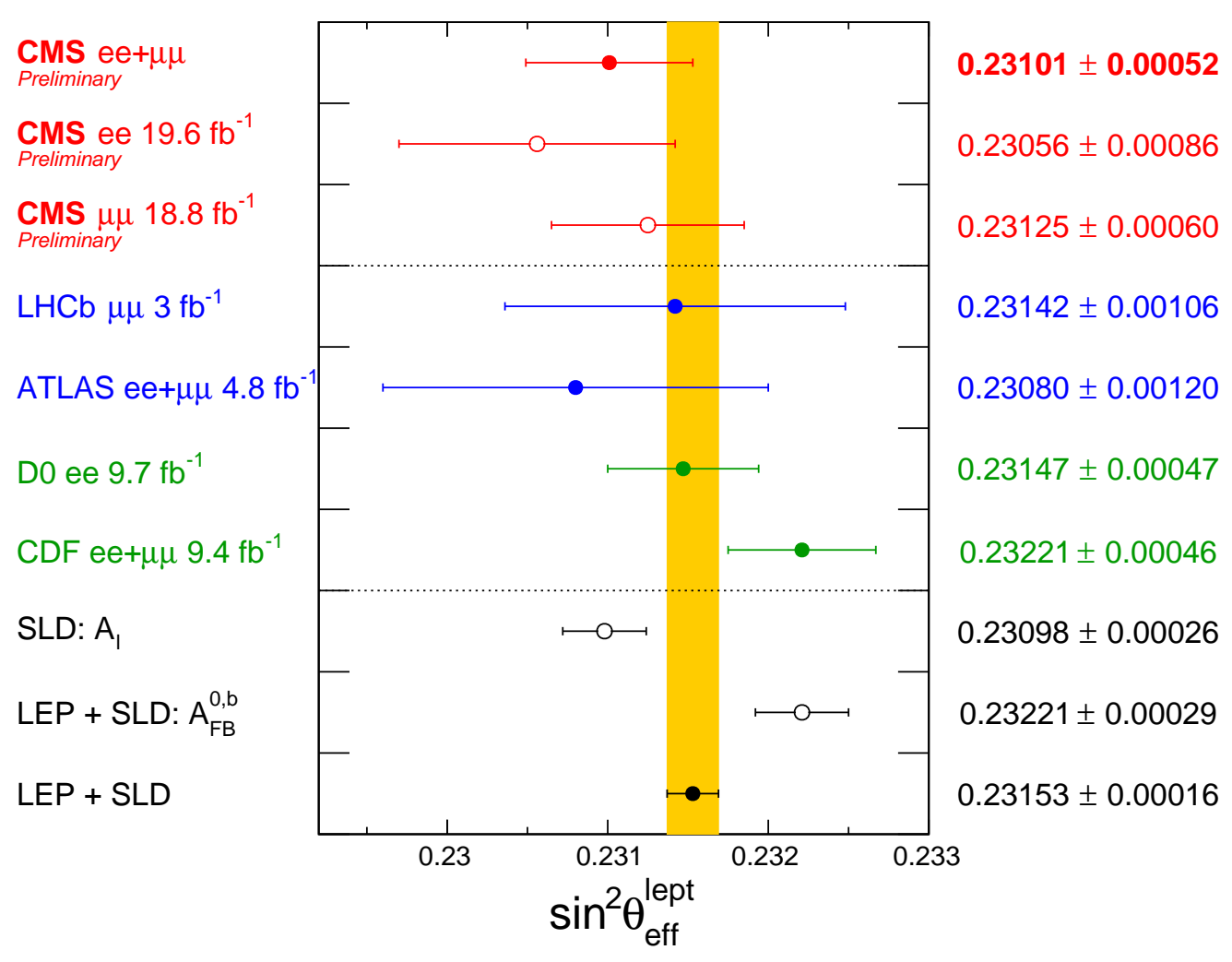

Figure 6: New CMS measurement of the electroweak mixing angle compared with other measurements at hadron and $\mathrm{e}^{+} \mathrm{e}^{-}$colliders.

the invariant mass of the dimuon system. In a similar study, the LHCb experiment had observed significant deviations with respect to theoretical predictions [16]. The new CMS measurement, while compatible with previous experimental results, is also in agreement with those theoretical predictions.

Searches for supersymmetry in CMS are now exploring new territory in the context of electroweak production. This is possible thanks to the substantial increase in collected luminosity at $\sqrt{s}=13 \mathrm{TeV}$ in 2016. Of particular interest is the comprehensive set of studies reported in [17]. Figure 8-top shows the example of the search for $\tilde{\chi}_{1}^{0} \tilde{\chi}_{1}^{0}$ production assuming that the neutralino decays via the neutral channels $\tilde{\chi}_{1}^{0} \rightarrow \mathrm{H}+$ missing energy and/or $\tilde{\chi}_{1}^{0} \rightarrow \mathrm{Z}+$ missing energy. The search is quantitatively interpreted in the context of a GMSB model with unknown (but comple-

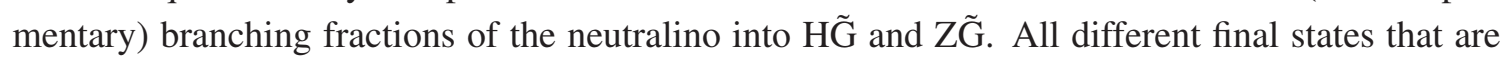
explored contribute effectively to exclude a neutralino on this model for masses up to $650 \mathrm{GeV}$ or so, independently of branching fraction assumptions.

Current LHC strategies in the search for dark matter are giving more weight to the information from searches for mediators using visible channels. An interesting example is the search for new physics in high-mass dijet production using an angular analysis [17]. The analysis is sensitive to new large-width resonances via relative excesses in central production, and thus complements the search for narrow resonances [18]. Figure 8-bottom shows this complementarity: both analyses are 


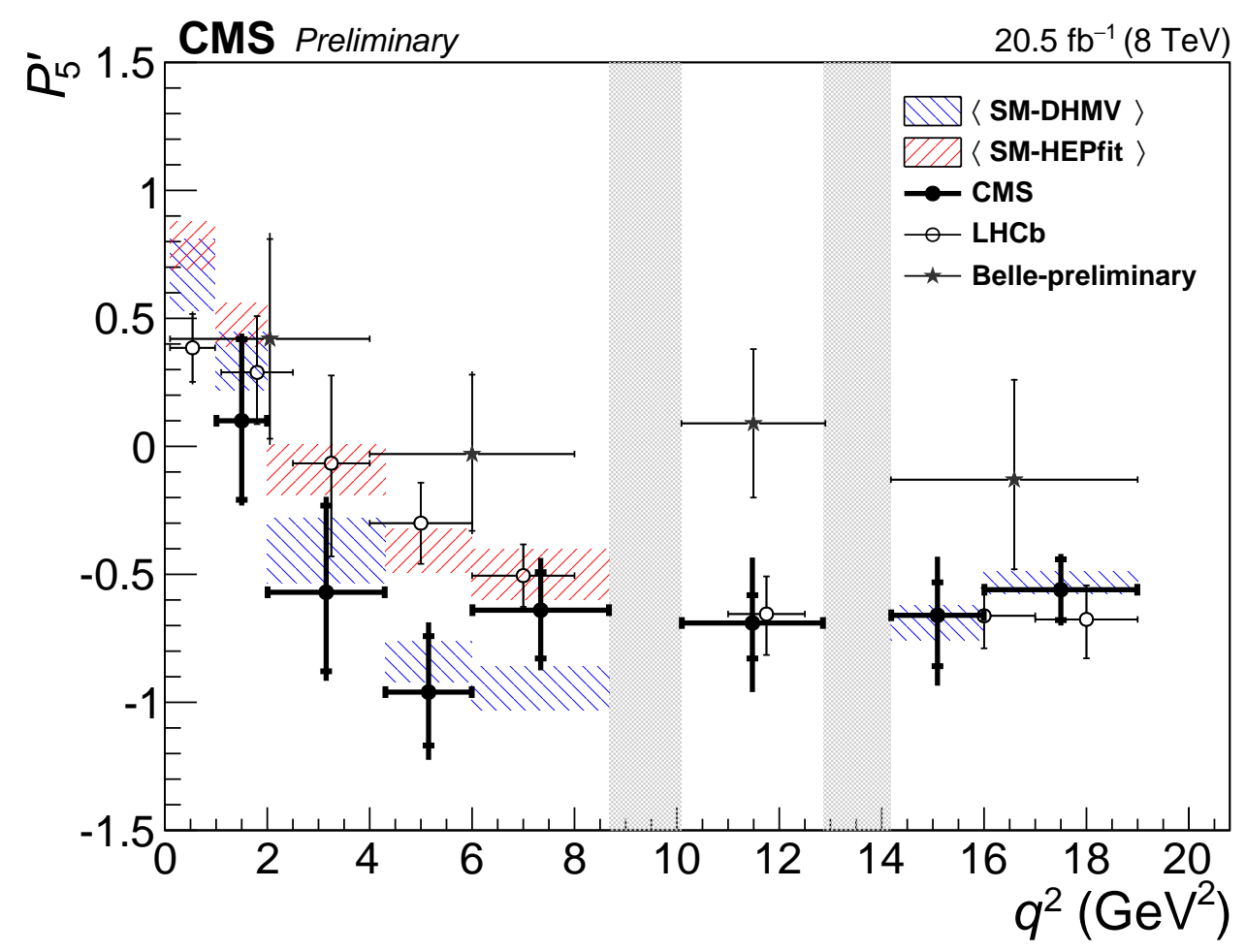

Figure 7: Measured values of the P5' coefficient in the angular analysis of the $\mathrm{B}^{0} \rightarrow \mathrm{K}^{* 0} \mu^{+} \mu^{-}$decay performed by CMS [14], compared with previous experimental results and several predictions. Values are measured as a function of the square of the invariant mass of the dimuon system $\left(q^{2}\right)$. The vertical shaded bands correspond to the excluded regions around the $\mathrm{J} / \psi$ and $\psi^{\prime}$ resonances.

sensitive to a significant part of the available parameter space for dark-matter mediators in the $\mathrm{TeV}$ range.

\section{Acknowledgments}

The author wishes to thank all CMS Collaboration members, who are at the origin of the contributions and results summarized in this report. We congratulate our colleagues in the CERN accelerator departments for the excellent performance of the LHC. This work is partially supported by the Spanish Ministry of Economy and Competitiveness (MINECO) through the grant FPA201453938-C3-1-R and the Unidad de Excelencia María de Maeztu CIEMAT - Física de Partículas, grant MDM-2015-0509.

\section{References}

[1] CMS Collaboration, S. Chatrchyan et al., The CMS Experiment at the CERN LHC, JINST 3 (2008) S08004.

[2] A. Charkiewicz, CMS Upgrade Status Report, Tech. Rep. CERN-RRB-2017-033, CERN, Geneva, https://cds.cern.ch/record/2254120. 

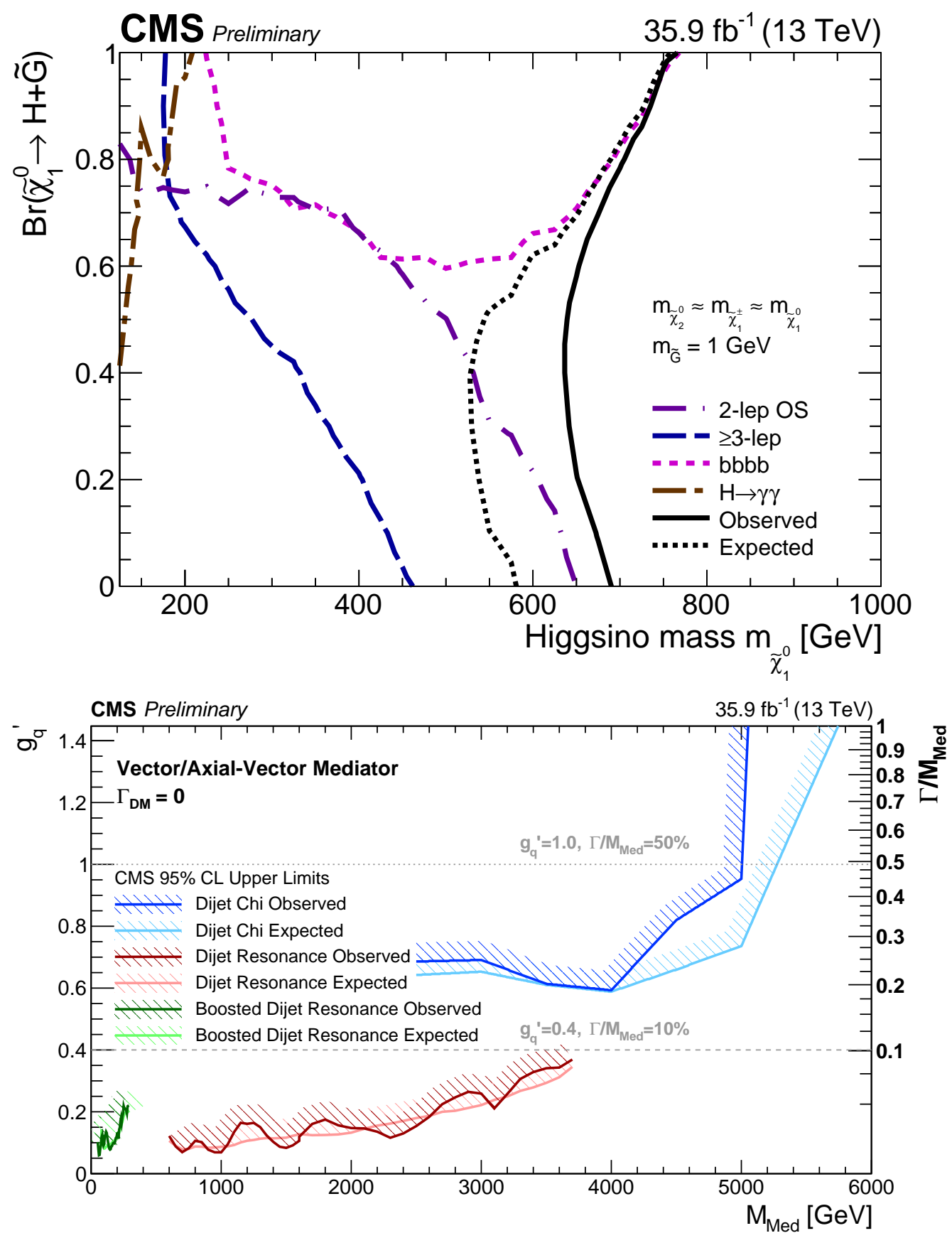

Figure 8: Top: observed exclusion contours at the $95 \% \mathrm{CL}$ in the $\left(B\left(\tilde{\chi}_{1}^{0} \rightarrow \mathrm{HG}\right), \mathrm{m}_{\tilde{\chi}_{1}^{0}}\right)$ plane for the GMSB model considered in [17]. The $4 \mathrm{~b}$ search drives the exclusion at large values of the branching fraction, while the Z-dilepton search and the multilepton search search are the most sensitive ones at lower values. Bottom: dark-matter related exclusion regions from CMS dijet searches [19]. The results are presented as a function of the quark coupling to the dark-matter mediator $\left(g_{q}^{\prime}\right)$ and the mediator mass $\left(\mathrm{M}_{\mathrm{Med}}\right)$. Searches for narrow dijet resonances [18] dominate the region of low couplings, while the search for deviations from new physics via dijet angular distributions constrains significantly and in a complementary manner the available phase space for mediator masses in the TeV range. 
[3] A. Dominguez, D. Abbaneo, K. Arndt, N. Bacchetta, A. Ball, E. Bartz et al., CMS Technical Design Report for the Pixel Detector Upgrade, Tech. Rep. CERN-LHCC-2012-016. CMS-TDR-11, https://cds.cern.ch/record/1481838.

[4] CMS Collaboration, CMS ECAL Laser monitoring up to 2017, $\pi^{0} / \eta \rightarrow \gamma \gamma$ spectrum and monitoring, ES calibration, . https://cds.cern.ch/record/2273269.

[5] T. Mc Cauley, "Large-mass di-muon event recorded by the CMS detector in 2017 (Run 2, 13 TeV)." Jul, 2017, https://cds.cern.ch/record/2272982.

[6] CMS Collaboration, A. M. Sirunyan et al., Measurements of properties of the Higgs boson decaying into the four-lepton final state in pp collisions at sqrt(s) $=13 \mathrm{TeV}, 1706.09936$.

[7] LHC Higgs Cross Section Working Group Collaboration, D. de Florian et al., Handbook of LHC Higgs Cross Sections: 4. Deciphering the Nature of the Higgs Sector, 1610.07922.

[8] CMS Collaboration, A. M. Sirunyan et al., Constraints on anomalous Higgs boson couplings using production and decay information in the four-lepton final state, 1707.00541.

[9] CMS Collaboration, A. M. Sirunyan et al., Observation of the Higgs boson decay to a pair of tau leptons, 1708.00373.

[10] CMS Collaboration, A. M. Sirunyan et al., Inclusive search for a highly boosted Higgs boson decaying to a bottom quark-antiquark pair, 1709.05543.

[11] CMS Collaboration, Search for Higgs boson pair production in the final state containing two photons and two bottom quarks in proton-proton collisions at $\sqrt{s}=13 \mathrm{TeV}$, Tech. Rep.

CMS-PAS-HIG-17-008, CERN, Geneva, https://cds.cern.ch/record/2273383.

[12] CMS Collaboration, A. M. Sirunyan et al., Observation of electroweak production of same-sign W boson pairs in the two jet and two same-sign lepton final state in proton-proton collisions at $\sqrt{s}=13$ $\mathrm{TeV}, 1709.05822$.

[13] CMS Collaboration, Measurement of the weak mixing angle with the forward-backward asymmetry of Drell-Yan events at $8 \mathrm{TeV}$, Tech. Rep. CMS-PAS-SMP-16-007, CERN, Geneva, https://cds.cern.ch/record/2273392.

[14] CMS Collaboration, Measurement of the $P_{1}$ and $P_{5}^{\prime}$ angular parameters of the decay $\mathrm{B}^{0} \rightarrow \mathrm{K}^{* 0} \mu^{+} \mu^{-}$ in proton-proton collisions at $\sqrt{s}=8 \mathrm{TeV}$, Tech. Rep. CMS-PAS-BPH-15-008, CERN, Geneva, https://cds.cern.ch/record/2256738.

[15] CMS Collaboration, V. Khachatryan et al., Angular analysis of the decay $B^{0} \rightarrow K^{* 0} \mu^{+} \mu^{-}$from $p p$ collisions at $\sqrt{s}=8 \mathrm{TeV}$, Phys. Lett. B753 (2016) 424-448, [1507.08126].

[16] LHCB Collaboration, R. Aaij et al., Angular analysis of the $B^{0} \rightarrow K^{* 0} \mu^{+} \mu^{-}$decay using $3 f b^{-1}$ of integrated luminosity, JHEP 02 (2016) 104, [1512. 044 42].

[17] CMS Collaboration, Combined search for electroweak production of charginos and neutralinos in pp collisions at $\sqrt{s}=13 \mathrm{TeV}$, Tech. Rep. CMS-PAS-SUS-17-004, CERN, Geneva, https://cds.cern.ch/record/2273907.

[18] CMS Collaboration, Searches for dijet resonances in pp collisions at $\sqrt{s}=13 \mathrm{TeV}$ using data collected in 2016., Tech. Rep. CMS-PAS-EXO-16-056, CERN, Geneva, https://cds.cern.ch/record/2256873.

[19] CMS Collaboration, Search for new physics with dijet angular distributions in proton-proton collisions at $\sqrt{s}=13 \mathrm{TeV}$ and constraints on dark matter and other models, Tech. Rep. CMS-PAS-EXO-16-046, CERN, Geneva, https://cds.cern.ch/record/2273455. 